\title{
BMJ Open Prevalence and predictors of road crash involvement among medical doctors in Malaysia: a cross-sectional study protocol
}

Aneesa Abdul Rashid (10 ,1,2,3 Navin Kumar Devaraj (10) ,1,3 Halidah Mohd Yusof, ${ }^{1}$ Fauzan Mustapha, ${ }^{1}$ Shaw Voon Wong, ${ }^{4}$ Ahmad Filza Ismail, ${ }^{5}$ Khairil Idham Ismail, ${ }^{2,6}$ Ahmad Munir Qureshi, ${ }^{7}$ Rusli Bin Nordin ${ }^{2,8}$

To cite: Rashid AA, Devaraj NK,

Mohd Yusof $\mathrm{H}$, et al.

Prevalence and predictors of road crash involvement among medical doctors in Malaysia: a cross-sectional study protocol. BMJ Open 2020;10:e037653. doi:10.1136/ bmjopen-2020-037653

- Prepublication history and additional material for this paper are available online. To view these files, please visit the journal online (http://dx.doi. org/10.1136/bmjopen-2020037653).

Received 11 February 2020 Revised 21 April 2020 Accepted 29 May 2020
Check for updates

(C) Author(s) (or their employer(s)) 2020. Re-use permitted under CC BY-NC. No commercial re-use. See rights and permissions. Published by BMJ.

For numbered affiliations see end of article.

Correspondence to Dr Aneesa Abdul Rashid; aneesa@upm.edu.my

\section{ABSTRACT}

Introduction Medical doctors are often subjected to long working hours with minimal rest in between the shifts. This has led to many fatal and non-fatal road crash involvement (RCl). This study aims to determine the prevalence and predictors of $\mathrm{RCl}$ among medical doctors in Malaysia. Methods and analysis This is a cross-sectional study among 375 Malaysian medical doctors who met the inclusion criteria. A predetermined self-administered questionnaires will be used to collect information regarding the sociodemographic, health status, workplace information, work commuting information, driving behaviour, history of $\mathrm{RCl}$, fatigue, sleep quality, mental health status and work engagement. The questionnaires consist of the following instruments: (1) sociodemographic, health status, workplace information, work commuting information, driving behaviour and history of $\mathrm{RCl}$; (2) Checklist of Individual Strength Questionnaire; (3) Pittsburgh Sleep Quality Index; (4) 21-item Depression Anxiety and Stress Scale; and (5) Utrecht's Work Engagement Scale. The data will be analysed using SPSS program V.24. Descriptive and inferential statistics will be used to determine the prevalence and predictors of $\mathrm{RCl}$. Ethics and dissemination This study protocol has received ethics approval from the Medical Research and Ethics Committee (MREC), Ministry of Health Malaysia (NMRR-18-3983-40609) and the Ethics Committee for Research Involving Human Subject, University Putra Malaysia (JKEUPM). Online written informed consent will be obtained from each study participant by the researchers. Results of the study will be disseminated through relevant journals and conferences. Trial registration number NCT04243291.

\section{INTRODUCTION}

Road crash involvement (RCI) and injuries are one of the major public health problems globally. The International Labour Organization reports that 2.2 million deaths are recorded every year, and 158000 are due to commuting accidents. Commuting accident is defined as 'any accident happening while travelling on a route between a place of residence to a place
Strengths and limitations of this study

- This nationwide study will determine the prevalence and predictors of road crash involvement (RCI) among Malaysian medical doctors.

- The outcome of this study will provide important information for preventative measures to address the $\mathrm{RCl}$ among medical doctors.

- The use of non-probability sampling will introduce selection bias into the study even though it is economical and logistically advantageous.

- The response rate will be partially influenced by the internet capability and information technology literacy of the participants.

of work: travelling on a journey made for any reason which is directly connected to employment; or travelling on a journey during any authorised recess'. ${ }^{12}$ In Europe, $15 \%$ of workrelated accidents are commuting accidents. ${ }^{3}$ In Malaysia, data on work-related accidents as reported by the Social Security Organisation showed that commuting accidents increased from 31314 in 2016 to 33319 accidents in 2017. ${ }^{4}$ Commuting accidents contributed almost half $(47.61 \%)$ of the total accidents in 2017. This shows an increasing trend since $2016 .{ }^{4}$ The trend also showed that the accidents are mostly due to travelling to and from work, before morning shift and within $5 \mathrm{~km}$ of travel distance, with motorcycle riders having the worse casualties. ${ }^{5}$

The issue of medical doctors and commuting accidents has recently been highlighted in the media and scientific writing, calling for urgent action to resolve the problem. ${ }^{6-8}$ Reports from the USA and Israel indicated that the prevalence of RCI among medical doctors, including those from the Emergency, Anaesthesiology, Medical, Surgical and Paediatric Departments, varies from $7.9 \%$ to 
$24.6 \% .^{9-12}$ A 1996 study among 70 junior doctors revealed that $44 \%$ had fallen asleep when stopped at a traffic light and $49 \%$ had fallen asleep at the wheels. Ninety per cent of these events occurred after long working hours (post call), and 20 out of 70 doctors were involved in RCI. ${ }^{9}$ Findings among US junior doctors also showed that long working hours, particularly extended shifts, led to more RCI. ${ }^{12}$ It is not just long hours but the time during which the doctors perform their duties also play a role; RCI is commonly reported after a night shift. ${ }^{11}{ }^{13}$ All the studies mentioned that the link to the RCI was sleep deprivation. ${ }^{9} 1112$ Nonetheless, it is worth mentioning that accidents still occur due to long working hours or night shift, which can be up to $15 \%$ of total accidents. ${ }^{9}$ Apart from sleep deprivation, working hours and time of work, other RCI related factors have also been proposed including fatigability. ${ }^{13}$ Doctors are known to work long hours, sometimes extending beyond 24 hours, resulting in sleep deprivation and fatigability. ${ }^{14-16}$ Fatigue and sleep deprivation have been studied extensively, especially among healthcare professionals, and have been reported to have a negative impact on their work and well-being. ${ }^{17}$ This includes reduced clinical judgement, impaired neurocognitive functioning and negative effect on the mood. Reduced attention and reaction time have been shown to have profound impact on driving of a motor vehicle. ${ }^{17}$ Sleep-related disturbance such as obstructive sleep apnoea, micro sleep and poor sleep hygiene are also said to affect the driving performance. ${ }^{18}$ After examining other contributors of RCI among medical doctors, gender, distance from work, number of dependent children, health status, exercise and use of medication were associated with RCI among healthcare and allied health professionals. ${ }^{19} 20$ The same studies also relate mental health factors, such as work satisfaction and job stress, on driving performance. ${ }^{1920}$ Local data pertaining to this issue is very limited. Ministry of Health Malaysia (MOH) data showed that 554 commuting accidents occurred between the years 2014 and 2016 among healthcare personnel. The distribution of commuting accidents, according to job category, revealed that $54 \%$ were nurses, followed by medical doctors $(6.3 \%)$, medical assistants (6.3\%), hospital attendants $(16.4 \%)$, drivers $(5.6 \%)$, food preparation attendants $(2.7 \%)$ and others $(9.4 \%) .{ }^{21}$ It is obvious that the proportion of doctors involved in RCI is not the highest among healthcare workers; however, doctor numbers are 3-4 times lower than staff nurses, combined with a possibility of under reporting. Also, as the country has heavily invested in training and employment of doctors, this figure will impact the economy and quality of healthcare delivery. ${ }^{22}$ A quick online 24 hours survey among Malaysian medical doctors with RCIs experience revealed that more than 440 of them who were admitted between 2009 and 2015 experienced RCI after long hours of work. ${ }^{23}$ However, this survey was carried out by medical non-governmental organisations (NGOs) with limitations in its study design, as it was one of the first quick surveys to explore such issue. This was a response of a few road crash fatalities among medical doctors due to long hours of work. Since then, this issue has been brought up by several other NGOs. Therefore, this suggests that the officially reported numbers is just the tip of the iceberg.

In terms of economic burden, if a medical doctor is involved in a bad or fatal accident, the implications are huge. To put this into perspective, the Malaysian Medical Council (MMC) in coordination with the Malaysian Qualifications Agency has ruled that every medical practitioner needs to complete a minimum of 5 years of undergraduate programme followed with 2 years of houseman ship training. The total fee for 5 years of undergraduate training in Malaysia ranges fromRM10 000-RM450 000. ${ }^{24}$ On top of this, the total emolument for a medical doctor ranges from RM4300-RM23 000 per month depending on the specified salary grades. ${ }^{22}$ Thus, losing a medical doctor will cause great financial burden to the economy, country and the families involved.

Due to the aforementioned issue, and the lack of published data, many NGOs have joined together with the help of researchers from local universities to explore and help understand this problem.

Findings of this study will be used to highlight the magnitude of the problem as well as to recommend and assist in developing appropriate intervention module to help doctors combat fatigue, and address factors related to commuting accidents and RCI.

\section{METHODS AND ANALYSIS}

\section{Study design}

Cross-sectional online questionnaire study.

\section{Study area}

Malaysia (nationwide).

\section{Study duration}

April 2020- May 2021.

\section{Study participants}

Malaysian Medical Association (MMA) members who are fully or partially registered with the MMC and their contacts. MMA is the main representative body for all registered medical practitioners in Malaysia. MMA members include senior and junior doctors, private and public, specialists and general practitioners, medical officers and house officers.

The eligibility criteria are based on the following:

\section{Inclusion criteria}

1. Malaysian citizen.

2. Full or partial registration with MMC.

3. Work a minimum of 6 months in the healthcare sector in Malaysia.

\section{Exclusion criteria}

1. Medical doctors currently on long leave.

2. Does not self-commute to work.

3. Those with psychiatric illness. 


\section{Sample size}

The prevalence of RCI among doctors is 7.9\%$24.6 \%{ }^{9-12}$ We choose the prevalence $(\mathrm{P})$ of $25 \%$ for cases of RCI as it yields the highest sample size, taking into account $95 \%$ confidence level and $5 \%$ margin of error. The formula for sample size calculation (single proportion) is as follows:

$$
\mathrm{n}=\mathrm{z}_{1-\alpha / 2}^{2} \mathrm{P}(1-\mathrm{P}) / \mathrm{d}^{2}
$$

Where $\mathrm{n}$ is the sample size, $\mathrm{P}$ is the prevalence of interest, $\mathrm{z}$ is the standard normal value corresponding to the desired confidence level $(\alpha)$ and $d$ the maximum error that is allowed (margin of error). Based on this formula, $\mathrm{z}=1.96$ (confidence level of $95 \%$ ), $\alpha=5 \%$ and $\mathrm{d}=0.5$. Considering $30 \%$ non-response or drop-out rate, the sample size is increased from 288 to 375 .

\section{Selection criteria}

We initially planned to use the systematic random sampling method to select the participants for this study based on the MMC Register. However, based on advice from the Medical Research and Ethics Committee (MREC) on the possible low response rate based on the previous experience, and possible long delay due to administrative issues, MREC have advised online methods and non-probability random sampling among MMA registered member list and their network. The sample will be doctors who are partially or fully registered with the MMC and fulfils the inclusion and exclusion criteria. Eligible participants will need to answer a questionnaire via an online survey link.

\section{Data collection}

This study will be in collaboration with the MMA that will recruit subjects along with other medical NGOs including the Islamic Medical Association of Malaysia, the lead NGO in this project. MMA will help in dissemination of information through their website as well as distribute/ email the questionnaires to all MMA members and their contacts. Participants will answer the self-administered questionnaire via the online link. The estimated time for participants to complete this questionnaire is $20-30 \mathrm{~min}$. The questionnaire will be administered only once to the subjects and the duration of the study is estimated to be 1 year. On completion of the questionnaire, the participants will have no further commitment to this research. However, participants will be informed if the study data is potentially useful to their well-being.

The questionnaire will be created by using Google Form and then will be saved on Google Drive. Then MMA will share the form to all of its members through email. All responses will be saved in the file stored in Google Drive. Once the sample size is acquired, the form will be closed and results will be downloaded for data analysis. The data collected will be recorded in another separate offline document after being retrieved from Google Form and all data in the Google Form will be destroyed.

\section{Data analysis}

Data entry and analysis will be undertaken using the SPSS program, V.24.0. Statistical significance is decided at $\mathrm{p}<0.05$ with $95 \%$ CI. Continuous variables will be summarised as means and SDs for normally distributed data; median and IQR for non-normal distribution. Categorical variables will be summarised as frequencies and percentages. Pearson $\chi^{2}$ tests will be employed to determine statistical significance of differences across categorical variables. Lastly, multiple logistic regression analyses will be undertaken to determine the strength of association between the outcome and factors of interest, adjusting for covariates or confounders. It also allows one to determine important factors affecting the outcome of interest and generating the final model for prediction. Multicollinearity and interactions will be examined to exclude redundant variables and to test for significant interactions among independent variables, respectively. Preliminary final model will be assessed using the following goodness-of-fit tests: Hosmer-Lemeshow test, classification table and area under receiver operating characteristics curve. Primary outcome will be the prevalence of RCI among medical doctors. Secondary outcomes will be associated factors contributing toward RCI that include sociodemographics, health status, workplace information, work commuting information, driving behaviour, involvement in road traffic accident, fatigue, sleep quality, mental health status and work engagement.

\section{Study instrument \\ Questionnaire / scoring}

A predetermined self-administered questionnaire (online supplementary appendix 1) will be used to measure the sociodemographics, health status, workplace information, work commuting information, driving behaviour, involvement in RCI, fatigue, sleep quality, mental health status and work engagement. All of the questionnaires are free for use as it is public domain, non-commercial and for academic purpose. The questionnaire is divided into five parts:

\section{Part A}

This is pertaining to the attributes of the respondents. Details include the sociodemographics, work information, health and lifestyle, and involvement in RCI and near misses. It is divided into six sections as follows:

- Section A consists of six questions on sociodemographics (age, ethnicity, gender, marital status and educational background).

- Section B consists of seven questions on health status (medical illness, exercise and smoking status).

- Section $\mathrm{C}$ consists of 20 questions on workplace information (length of employment, employer, current position and department, duration of work, work hours, work routine, napping and exposure to chemicals). 
- Section D consists of six questions on work commuting information (including mode of transportation, distance and time travelled). One question on a valid driver's licence.

- Section E is on driving behaviour. This has 11 questions (driving habits, speed of driving, occurrences of mind wandering, sudden outbursts, distraction, driving circumstances such as under alcohol influence, history of nodding off and falling asleep while driving, losing focus while driving, consuming substance before and while driving, and driving for fun).

- Section F is involvement in RCI. This consists of 24 questions (involvement in RCI, near miss accident, explaining the worse encountered accident, injury and its losses).

\section{Part B}

Online supplementary appendix 2 pertaining to the sleep quality using the Pittsburgh Sleep Quality Index (PSQI) ${ }^{25}$ The PSQI assesses the quality and patterns of sleep, differentiating 'poor' and 'good' sleep using a measurement of seven items: subjective sleep quality, sleep latency, sleep duration, habitual sleep efficiency, sleep disturbances, use of sleeping medication and daytime dysfunction over the last month. Scoring of answers is based on a 0-3 scale, whereby 3 reflects the negative extreme on the Likert Scale. The score for each component will be summed up as the Global PSQI score. A Global PSQI score of ' 5 ' or greater indicates a 'poor sleeper'.

\section{Part C}

Online supplementary appendix 3 pertaining to the level of fatigue using the Checklist of Individual Strength Questionnaire (CISQ) ${ }^{26}$ in English and Malay Language as obtained from a prior MIROS study. The CISQ is a 20 -item questionnaire designed to measure aspects of fatigue and asking respondents of their feelings during the preceding 2 weeks. It is a self-report and consists of four components identified via factor analysis for subjective experience of fatigue (eight items), concentration (five items), motivation (four items) and level of physical activity (three items). The total scale has a Cronbach's alpha of 0.93 , and is scored using the 7-point Likert scale. Higher scores indicate higher degree of fatigue, more concentration problems, reduced motivation and less physical activity. A composite CISQ score that ranges between 20 and 140 is constructed by summing up individual scores from all four components to capture the subjective sensation of fatigue and reduced functioning (ie, concentration, motivation and activity level). Case classification is obtained by implementing a cut-off CISQ point of $>76$; those who scored more than 76 are designated as probable fatigue case. This cut-off value was established in a different pilot study using defined samples with differences in the fatigue level. This questionnaire has been validated and has been used for other studies. Therefore, no validation process is required for this questionnaire.

\section{Part $D$}

Online supplementary appendix 4 has two versions. The original version has 42 questions, and the shorter version is the Depression Anxiety and Stress Score consisting of 21 items (DASS 21). The scores will categorise individuals into low, moderate or severe in each domain. Both questionnaires have a Cronbach's alpha score of 0.96-0.97 for DASS-Depression, 0.84-0.92 for DASS-Anxiety and 0.900.95 for DASS-Stress. Studies have found this tool to be a good tool to assess general and clinic based population. For this study, the DASS 21 will be used as it is shorter, and more convenient.$^{27} 28$

\section{Part E}

Online supplementary appendix 5 The Utrecht's Work Engagement Scale (UWES) assesses positive psychological well-being of employees while at work. ${ }^{29}$ It consists of 17 items, categorised under the dimensions of vigour, dedication and absorption. Each item is scored on a 7-point scale ranging from 0 (never) to 6 (everyday). Cronbach's alpha score for vigour ranged between 0.75 and $0.85,0.86$ and 0.90 for dedication and 0.82 and 0.88 for absorption. Higher scores indicate higher work engagement and lower burnout rates.

\section{Validation of questionnaires}

All these questionnaires have already been validated in Malaysia except for the sociodemographics, health status, workplace information, work commuting information, driving behaviour and involvement in road traffic accident. Validation for this section will be done using face and content validation. Face validation will be done with $10 \%$ calculated sample size and content validation will be done with expert panel consisting of two family medicine specialists and three public health medicine specialists. PQSI, CISQ DASS (Depression, Anxiety and Stress)-21 and UWES will be validated using a small sample of Malaysian medical doctors. Postvalidation changes will be made to the questionnaire before recruitment.

\section{Patient and public involvement statement \\ No patients involved.}

\section{Ethical considerations}

This study has received the approval for ethics clearance from the MREC, MOH Malaysia (NMRR-18-398340609) and the Ethics Committee for Research Involving Human Subjects, University Putra Malaysia (JKEUPM). Written informed consent will be obtained from each study participant and it will be stated at the beginning of the questionnaire. They will not be required to sign into an account in order to fill in the survey. They will also be told the right not to respond to the questions if they do not want to. All data will be kept confidential for research purposes only and will not be disclosed to any third parties. For publication, all personal information will be deidentified to protect the identity of the participants. Google Form responses are stored in a worksheet that can only be accessed through a Google login; only 
two of the researchers have access to this. The account is password protected. Data collected will be recorded in another separate offline document after being retrieved from Google Form and all data in the Google Form will be destroyed. Research data will only be accessed by the two researchers, above. The data will be stored for at least 7 years from the date of final publication.

\section{Dissemination}

Results of this study will be disseminated by publication through peer-reviewed professional and scientific journals. The participants' data will be kept confidential and will not be shared with the public. If there are requests for data sharing for appropriate research purposes, this will be considered on an individual basis after the trial completion and after the publication of the primary manuscripts.

\section{Author affiliations}

${ }^{1}$ Department of Family Medicine, Faculty of Medicine and Health Sciences, Universiti Putra Malaysia, Serdang, Malaysia

${ }^{2}$ Department of Research, Islamic Medical Association Malaysia, Cheras, Malaysia ${ }^{3}$ Malaysian Research Institute On Ageing (MYAGEING), Universiti Putra Malaysia, Serdang, Selangor, Malaysia

${ }^{4}$ Mechanical and Manufacturing Engineering, Universiti Putra Malaysia, Serdang, Malaysia

${ }^{5}$ Department of Community Medicine, Universiti Sains Malaysia - Kampus Kesihatan, Kubang Kerian, Malaysia

${ }^{6}$ Non-Communicable Disease (NCD) Unit, Putrajaya District Health Office, Ministry of Health Malaysia, Putrajaya, Malaysia

${ }^{7}$ Visiting Faculty, Al-Shifa School of Public Health, Rawalpindi, Pakistan

${ }^{8}$ Faculty of Health and Medical Sciences, School of Medicine, Taylor's University,

Subang Jaya, Malaysia

Acknowledgements The authors would like to thank the Ministry of Education Malaysia for funding this study. Grant no: FRGS/1/2018/SKK01/UPM/03/1.

Contributors Study design: AAR, NKD, RBN, AFI, AMQ, KII and SVW. Drafting the work and revising critically for important intellectual content: AAR, NKD, FM, RBN and HMY. Final approval of the version to be published: AAR, NKD, RBN, AFI, AMQ, KII, SVW, FM and HMY.

Funding This study is funded by the Ministry of Education Malaysia and Universiti Putra Malaysia (UPM). Grant no: FRGS/1/2018/SKK01/UPM/03/1.

Competing interests None declared.

Patient consent for publication Not required.

Provenance and peer review Not commissioned; externally peer reviewed.

Open access This is an open access article distributed in accordance with the Creative Commons Attribution Non Commercial (CC BY-NC 4.0) license, which permits others to distribute, remix, adapt, build upon this work non-commercially, and license their derivative works on different terms, provided the original work is properly cited, appropriate credit is given, any changes made indicated, and the use is non-commercial. See: http://creativecommons.org/licenses/by-nc/4.0/.

\section{ORCID iDs}

Aneesa Abdul Rashid http://orcid.org/0000-0002-7944-1364

Navin Kumar Devaraj http://orcid.org/0000-0002-9081-2162

\section{REFERENCES}

1 OECD. OECD glossary of statistical terms - commuting accident definition, 2017.
2 Beres E. Recording and notification of occupational accidents and diseases. 96. Geneva: International Labour Office, 1996.

3 Rusli Bin N, Nordin RB. Rising trend of work-related commuting accidents, deaths, injuries and disabilities in developing countries: a case study of Malaysia. Ind Health 2014;52:275-7.

4 PERKESO. Yearly Report [Internet]. Official Website Social Security Organisation (SOCSO). Available: https://www.perkeso.gov.my/index. $\mathrm{php} / \mathrm{ms} /$ laporan/laporan-tahunan

5 Hoe V. Commuting accidents in Malaysia: are we doing enough? Occup Environ Med 2014;71(Suppl 1):A94.

6 The Star Online. "Ensure wellbeing and welfare of healthcare staff" - Nation, 2017. Available: https://www.thestar.com.my/news/nation/ 2017/05/14/ensure-wellbeing-and-welfare-of-healthcare-staff/

7 Kaur Cheema R. Malaysian doctors at risk on the roads post-call [Internet], 2017. Available: https://today.mims.com/malaysiandoctors-at-risk-on-roads-post-call

8 Fruchtman Y, Moser AM, Perry ZH. Fatigue in medical residents-lessons to be learned. Med Lav 2011;102:455-63.

9 Marcus CL, Loughlin GM. Effect of sleep deprivation on driving safety in housestaff. Sleep 1996;19:763-6.

10 Frunchtman Y, Moser A, Perry M. Fatigue in medical residents lessons to be learned.

11 Steele MT, Ma OJ, Watson WA, et al. The occupational risk of motor vehicle collisions for emergency medicine residents. Acad Emerg Med 1999;6:1050-3.

12 Barger LK, Cade BE, Ayas NT, et al. Extended work shifts and the risk of motor vehicle crashes among interns. N Engl J Med 2005;352:125-34.

13 McClelland L, Holland J, Lomas J-P, et al. A national survey of the effects of fatigue on trainees in anaesthesia in the UK. Anaesthesia 2017;72:1069-77.

14 Jeffers $R$, Jeys $L$. Tired surgical trainees: unfit to drive but fit to operate? BMJ 2002;324:173a.

15 Millner PA. Tired surgical trainees. BMJ 2002;324:1154b.

16 Williams M. Tired surgical trainees. Sleep deprivation affects psychomotor function. BMJ 2002;324:1154.

17 Owens JA. Sleep loss and fatigue in healthcare professionals. $J$ Perinat Neonatal Nurs 2007;21:92-100.

18 Pandi-Perumal SR, Verster JC, Kayumov L, et al. Sleep disorders, sleepiness and traffic safety: a public health menace. Braz $J$ Med Biol Res 2006;39:863-71.

19 Kirkcaldy B, Trimpop R, Cooper CL. Working hours, job stress, work satisfaction, and accident rates among medical practitioners and allied personnel. Int J Stress Manag 1997;4:79-87.

20 Taylor AH, Dorn L. Stress, fatigue, health, and risk of road traffic accidents among professional drivers: the contribution of physical inactivity. Annu Rev Public Health 2006;27:371-91.

21 New Straits Times. Call for greater wellbeing of doctors and medical staff [Internet]. Available: https://www.nst.com.my/opinion/ letters/2017/06/247172/call-greater-wellbeing-doctors-andmedical-staff

22 Afterschool.my. Cost of studying medicine in Malaysia [Internet], 2016. Available: http://afterschool.my/articles/cost-of-studyingmedicine-in-malaysia

23 Aneesa AR, Suhazeli A, Nur Asyikin M, et al. Post call MVA survey: an 18 hour online survey. 17th Islamic Association of Malaysia (IMAM) Annual Scientific Conference (ASC), Penang, Malaysia, 2015.

24 World Health Organization Western Pacific Region. Human resources for health country profiles. Malaysia, 2014: 1-60.

25 Buysse DJ, Reynolds CF, Monk TH, et al. The Pittsburgh sleep quality index (PSQI): a new instrument for psychiatric research and practice. Psychiatry Res 1988;28:192-213.

26 Beurskens AJ, Bültmann U, Kant I, et al. Fatigue among working people: validity of a questionnaire measure. Occup Environ Med 2000;57:353-7.

27 Lovibond PF, Lovibond SH. The structure of negative emotional states: scales (DASS) with the Beck depression inventory. Behav Res Ther 1995;33:335-43.

28 Parkitny L, McAuley J. The depression anxiety stress scale (DASS). $J$ Physiother 2010;56:204.

29 Schaufeli WB, Bakker AB, Salanova M. The measurement of work engagement with a short questionnaire. Educ Psychol Meas 2006;66:701-16. 